\section{UNISIM: A text-editing BASIC program for creation and modification of sequential disk files}

\author{
NEIL R. SHAPIRO, ROBERT A. ROSELLINI, \\ and JOSEPH P. DeCOLA \\ State University of New York, Albany, New York 12222
}

The present program provides a sophisticated means for data entry and editing, permitting the microprocessor user to edit any disk file saved in ASCII format using the powerful command set of the UNIVAC 1100-series text editor.

When the researcher must rely on main-frame computers for data analysis, this program serves to complement any of several intelligent terminal programs now available. These programs, such as the one recently published by Kirk (1980), permit the microcomputer user to transfer disk files to a main-frame computer or other nonlocal facility. In this context, the present program may be used to create and edit disk elements that may be transferred to the main frame via such intelligent terminal programs. Thus, the program provides an inexpensive and efficient alternative to using cardpunch or main-frame editing facilities. Further, since intelligent terminal programs often allow bidirectional data transfer, elements can be brought back from the main frame, stored on disk, and edited to make them suitable for entry into local microcomputer programs. (An example is given in the "Applications" section.)

System Requirements. This program is written according to the syntax of Radio Shack Level II Disk BASIC 1.1, which requires at least TRSDOS 2.1. Differences between the BASIC language and others are the allowable variable names, permissibility of multiple statement lines, and the specific format of string manipulation commands. The program occupies approximately $9 \mathrm{~K}$ bytes of memory and is compatible with either a $32 \mathrm{~K}$ - or $48 \mathrm{~K}$-byte system.

Program Description. The program was designed with commands similar to the UNIVAC 1100-series text editor (UNISIM is short for UNIVAC text editor simulation). There are several reasons for choosing such a design: (1) The UNIVAC text editor is quite powerful, in that it provides the user with a wide variety of element manipulation commands; (2) each command has a well chosen mnemonic specification; and (3) as a widely used implementation, many users already familiar with the UNIVAC editor will need little additional study to utilize this program.

The program functions in three modes: input, insert, and edit. Input mode is used to create an element on a

This work was supported by NSF Grant BNS-7820678 to the second author. user-specified disk drive and to add lines to the end of an element. In insert mode, up to 100 lines may be added between any 2 existing lines. Provisions are also made for merging two separate elements.

A variety of editing commands are provided, each consisting of a one- to five-letter mnemonic. The most basic of these are the print commands, which allow the user to display on the CRT screen or the line printer any line or series of lines. String manipulation commands allow the user to add a variable amount of information to any position in the line ("append column" and "append line"), to string two lines together ("append to end"), to change the first or all occurrences of a substring in a line ("change" and "change globally"), or to change all subsequent occurrences of the string in the element ("change all"). Moreover, the character set represented on the keyboard is extended to the entire ASCII set by allowing one to change, or to change something to, a character by entering its decimal ASCII code.

One may delete lines, mark lines for deletion, or, conversely, unmark them. Similarly, columns in a line may be specified for deletion or may be written over. Line manipulations include "move lines" and "ditto" (copy). Informational commands allow the user to determine line length, string locations both within a line ("where's $x$ ") and among lines ("locate"). A transformation command allows the user to enter an algebraic expression and to have it executed on any number of lines in the element.

The complete instruction set is too large for an exhaustive list to be presented here, but other commands that should be of interest include the repeat and macro facilities. These two commands allow the user to repeat a single command a number of times automatically or to create a "macro" series of commands to be executed sequentially and to be saved for execution at any point during the editing session. The user can also examine the disk directory and create an index of the contents of elements on a specific diskette. However, these two commands must be supported by either a NEWDOS+ or a NEWDOS80 disk operating system.

When using either of these two operating systems in conjunction with a lowercase driver, UNISIM permits the entry of mixed text. To facilitate the use of the editor with lowercase, it will accept commands in the upper- or lowercase mode.

Applications. As already mentioned, UNISIM provides a useful adjunct to intelligent terminal programs for creating elements to be transferred to a main frame. Additionally, the program provides a means to edit elements recovered from the main-frame computer so that they may be used with microcomputer programs. For example, we have found it useful to recover means from the output of canned statistical programs via the intelligent terminal program and to edit these means 
using UNISIM so that they appear in a consistent lexicographic order. Once so ordered, they can be saved on diskette and analytical comparisons may then be performed via microcomputer programs such as $\mathrm{ANCOM}^{1}$ (Shapiro \& Rosellini, 1980). This is particularly valuable when the designs being analyzed involve several repeated measures, since in such cases there are often a large number of means that would otherwise need to be entered by hand.

UNISIM may also be used to edit other BASIC programs that are stored in ASCII format. The advantages this program offers over resident BASIC editors include the abilities to change strings throughout the program, copy lines, move lines, renumber portions of the program, search for strings (variables), and so on.

Availability. A program listing, an operations manual with examples of the use of each command, and a copy of the program on a minifloppy diskette can be obtained from the second author. To cover mailing, reproduction, and diskette cost, please include $\$ 15$.

\section{REFERENCES}

KIRK, R. C. HTERM/CIM and HTERM/BAS: "Smart" terminal enabling programs for the TRS-80 microcomputer. Behavior Research Methods \& Instrumentation, 1980, 12, 71-72.

Shapiro, N. R., \& Rosellini, R. A. ANCOM: A BASIC program for analytical comparisons among means. Behavior Research Methods \& Instrumentation, 1980, 12, 633.

\section{NOTE}

1. While the first version of ANCOM (as described in Shapiro \& Rosellini, 1980) required data to be entered interactively, the current version will input data either interactively or from diskette.

(Accepted for publication July 7, 1981.) 